\title{
Elevated expression of the nuclear export protein, Crm1 (exportin 1), associates with human oesophageal squamous cell carcinoma
}

\author{
PAULINE J. VAN DER WATT ${ }^{1}$, WIDAAD ZEMANAY ${ }^{1}$, DHIRENDRA GOVENDER ${ }^{2}$, \\ DENVER T. HENDRICKS ${ }^{1}$, M.I. PARKER ${ }^{1,3}$ and VIRNA D. LEANER ${ }^{1}$
}

\begin{abstract}
${ }^{1}$ Division of Medical Biochemistry, Institute of Infectious Disease and Molecular Medicine, Faculty of Health Sciences, University of Cape Town; ${ }^{2}$ Division of Anatomical Pathology, Faculty of Health Sciences, University of Cape Town, NHLS-Groote Schuur Hospital; ${ }^{3}$ International Centre for Genetic Engineering and Biotechnology (ICGEB), Cape Town Component, Cape Town, South Africa
\end{abstract}

Received February 13, 2014; Accepted April 29, 2014

DOI: $10.3892 /$ or.2014.3231

\begin{abstract}
The nuclear export receptor, $\mathrm{Crm1}$ (exportin 1), is involved in the nuclear translocation of proteins and certain RNAs from the nucleus to the cytoplasm and is thus crucial for the correct localisation of cellular components. Crm1 has recently been reported to be highly expressed in certain types of cancers, yet its expression in oesophageal cancer has not been investigated to date. We investigated the expression of Crm1 in normal and tumour tissues derived from 56 patients with human oesophageal squamous cell carcinoma and its functional significance in oesophageal cancer cell line models. Immunohistochemistry revealed that $\mathrm{Crm} 1$ expression was significantly elevated in oesophageal tumour tissues compared to normal tissues and its localisation shifted from predominantly nuclear to nuclear and cytoplasmic. Real-time RT-PCR revealed that $\mathrm{Crm} 1$ expression was elevated at the mRNA level. To determine the functional significance of elevated Crm1 expression in oesophageal cancer, its expression was inhibited using siRNA, and a significant decrease in cell proliferation was observed associated with G1 cell cycle arrest and the induction of apoptosis. Similarly, leptomycin B (LMB) treatment resulted in the effective killing of oesophageal cancer cells at nanomolar concentrations. Normal oesophageal epithelial cells, however, were much less sensitive to Crm1 inhibition with siRNA and LMB. Together, this study reveals that $\mathrm{Crm} 1$ expression is increased in oesophageal cancer and is required for the proliferation and survival of oesophageal cancer cells.
\end{abstract}

Correspondence to: Professor Virna Leaner, Division of Medical Biochemistry, Faculty of Health Sciences, University of Cape Town, Observatory, Cape Town 7925 South Africa

E-mail: virna.leaner@uct.ac.za

Key words: Crm1, nuclear transport, oesophageal cancer, apoptosis, leptomycin B

\section{Introduction}

Oesophageal cancer is the eighth most common cancer worldwide and the sixth most common cause of death from cancer (1). It is three to four times more common among males than females (2). Survival rates are poor, as patients with oesophageal cancer usually present with disease that is advanced and has already metastasised at the time of initial diagnosis. Oesophageal squamous cell carcinoma (OSCC) is the predominant histological subtype, comprising approximately $70 \%$ of cases. This cancer arises in the middle and lower third of the oesophagus. OSCC is particularly prevalent in the developing world, where highest incidence rates are in Southern and Eastern Africa and Eastern Asia (2). Risk factors include poor nutritional status, drinking beverages at high temperatures, smoking and alcohol consumption (2). However, the molecular basis of OSCC is still largely unknown. The identification of new molecular biomarkers would be of great importance for the early detection of the disease. Moreover, identification of molecular targets for therapeutic drugs would result in better treatment options for OSCC patients.

$\mathrm{Crm} 1$ (the chromosome region maintenance 1 protein or exportin 1) is a protein that has recently been identified as being highly expressed and playing a functional role in several types of cancers. Crm1 is a member of the karyopherin $\beta$ protein family and the major nuclear export receptor in the cell (3). It mediates the nuclear export of cargo proteins and certain RNAs from the nucleus into the cytoplasm, across the nuclear pore complex (NPC), thus facilitating the appropriate subcellular localisation of target proteins and RNAs. Crm1 has a broad substrate range, recognising cargo proteins that carry a leucine-rich nuclear export signal (NES) (4). Since cargo proteins include various transcription factors, cell cycle proteins and signalling proteins, for example, p53 (5) and Akt1 (6), it would appear that many of the integral processes in the cell depend on appropriate $\mathrm{Crm} 1$ expression and function.

Recent studies have reported that the expression of $\mathrm{Crm} 1$ is altered in cancer, with elevated Crm1 levels reported in cervical cancer (7), ovarian cancer (8), osteosarcoma (9), 
glioma (10), pancreatic cancer (11), gastric cancer (12) and multiple myeloma (13) compared to normal tissue. We previously identified increased Crm1 expression in transformed fibroblasts compared to normal fibroblasts, suggesting that the increased expression of $\mathrm{Crm} 1$ is a feature of the transformed phenotype (7). Furthermore, elevated Crm1 expression appears to be necessary for the survival of cancer cells, as we showed that its inhibition using siRNA resulted in cervical cancer cell death via apoptosis (7). Moreover, Crm1 inhibition using leptomycin B (LMB) has been well documented to have an anticancer effect both in vitro and in vivo (14). Although LMB failed clinical trials, studies are currently underway in an attempt to develop more effective inhibitors of Crm1 for cancer therapy $(15,16)$.

In the present study, we examined the expression and functional relevance of $\mathrm{Crm} 1$ expression in oesophageal squamous cell carcinoma. We found that Crm1 levels are elevated in oesophageal tumours compared to normal epithelium and that its cellular localisation is also altered. We also found that $\mathrm{Crm} 1$ is required for the proliferation and survival of oesophageal cancer cells, supporting its use as a potential target for anticancer drugs.

\section{Materials and methods}

Tissue sections. Immunohistochemical examination of Crm1 was performed on archived paraffin-embedded tissue sections of matched normal and cancer tissues obtained from 56 patients with oesophageal squamous cell carcinoma (Groote Schuur Hospital, Cape Town, South Africa). Immunohistochemistry was performed using standard procedures. Briefly, slides were heat-fixed for $10 \mathrm{~min}$, deparaffinised and rehydrated, followed by antigen retrieval by incubation in $10 \mathrm{mM}$ EDTA in a pressure cooker for $2 \mathrm{~min}$. Sections were blocked for endogenous peroxidase activity by submerging the slides in hydrogen peroxide for $20 \mathrm{~min}$ and were then incubated at room temperature with a 1:20 dilution of goat serum (Dako, Glostrup, Denmark) for $30 \mathrm{~min}$. Slides were next incubated with a 1:500 dilution of Crm1 primary antibody (sc-5595; Santa Cruz Biotechnology, Santa Cruz, CA, USA) for $1 \mathrm{~h}$ at room temperature. A negative control was included where no primary antibody was used. The Dako REAL ${ }^{\mathrm{TM}}$ EnVision ${ }^{\mathrm{TM}} / \mathrm{HRP}$ rabbit/mouse detection system was subsequently used to detect Crm1 expression, according to the manufacturer's instructions. The intensity and localisation of Crm1 staining were evaluated and scored independently by a pathologist. Crm1 expression was noted in nuclear and cytoplasmic cellular compartments and thus a separate scoring for Crm1 expression was performed in each case. For nuclear staining, the Allred system for oestrogen receptor was used (17), where the staining intensity was scored as 0 (negative), 1 (weak), 2 (moderate), or 3 (strong). The percentage of positive cells was not included in the scoring criteria as in all cases the proportion of positive cells was $>75 \%$. For cytoplasmic staining the following criteria were used: 0 (negative), 1 (weak), 2 (moderate), or 3 (strong) (18).

Quantitative real-time RT-PCR. For the analysis of Crm1 mRNA expression, RNA was isolated from matched normal and tumour tissue biopsies obtained from 22 patients with oesophageal squamous cell carcinoma (Groote Schuur
Hospital and Tygerberg Hospital, Cape Town, South Africa). For cDNA synthesis, $1 \mu \mathrm{g}$ RNA was reverse transcribed using ImProm-II ${ }^{\mathrm{TM}}$ Reverse Transcriptase (Promega, Madison, WI, USA). Quantitative real-time PCR was performed using the KAPA SYBR qPCR kit (KAPA Biosystems, Cape Town, South Africa) using the following primers: Crm1 (F 5'-GCA CCT CTT GGA CTG AAT CG-3' and R 5'-AAG CGA CAG CAC ACA CAC AC-3'), $\beta$-glucuronidase (F 5'-CTC ATT TGG AAT TTT GCC GAT T-3' and R 5'-CCG AGT GAA GAT CCC CTT TTT A-3') and cyclophilin D (F 5'-TGA GAC AGC AGA TAG AGC CAA GC-3' and R 5'-TCC CTG CCA ATT TGA CAT CTT C-3'), where $\beta$-glucuronidase and cyclophilin D were used to normalise for Crm1 expression. The StepOne Real-time PCR System (Applied Biosystems, USA) was used. The comparative threshold cycle $\left(\mathrm{C}_{\mathrm{T}}\right)$ method (19) was used for the calculation of expression fold change between samples.

Cell culture. Oesophageal carcinoma cell lines, WHCO1, WHCO5 and WHCO6, were originally established from South African patients with oesophageal squamous cell carcinoma and were provided by Dr R. Veale (20). Oesophageal carcinoma cell lines, KYSE30, KYSE70, KYSE150, KYSE180, KYSE420 and KYSE450, were either obtained from DSMZ (Berlin, Germany) or were a gift from Professor Y. Shimada (21). Cells were maintained in Dulbecco's modified Eagle's medium (DMEM), supplemented with penicillin $(100 \mathrm{U} / \mathrm{ml})$, streptomycin $(100 \mu \mathrm{g} / \mathrm{ml})$ and $10 \%$ fetal calf serum (Gibco, Paisley, Scotland). Normal hTERT-immortalised human oesophageal keratinocytes, EPC2-hTERT, were a gift from Professor A.K. Rustgi (University of Pennsylvania, Philadelphia, PA, USA). The cells were maintained in keratinocyte growth medium supplemented with $1 \mathrm{ng} / \mathrm{ml} \mathrm{EGF}$ and $50 \mu \mathrm{g} / \mathrm{ml}$ pituitary extract. All cells were cultured at $37^{\circ} \mathrm{C}$ in a humidified atmosphere of $5 \% \mathrm{CO}_{2}$.

Materials. For the inhibition of gene expression, Crm1 siRNA was used (sc-35116; Santa Cruz Biotechnology). Control siRNA-A consisting of a scrambled sequence (sc-37007; Santa Cruz Biotechnology) was used as a non-silencing control. Leptomycin B (LMB), an inhibitor of Crm1 activity, was obtained from Sigma (St. Louis, MO, USA) and stored as a $10.2 \mu \mathrm{M}$ stock in methanol.

Protein harvest and western blot analysis. Cells in culture were grown to $80 \%$ confluency and lysed on ice in RIPA buffer (10 mM Tris-Cl, pH 7.4, $150 \mathrm{mM} \mathrm{NaCl}, 1 \%$ deoxycholate, $0.1 \%$ SDS, $1 \%$ Triton X-100 and $1 \mathrm{X}$ Complete Protease Inhibitor Cocktail (Roche, Basel, Switzerland). Western blot analyses were performed using the rabbit anti-Crm1 (H-300) (sc-5595) and rabbit anti- $\beta$-tubulin ( $\mathrm{H}-235$ (sc-9104) antibodies (Santa Cruz Biotechnology).

Immunofluorescence. For immunofluorescence analysis of Crm1, cells were plated on coverslips and fixed with $4 \%$ paraformaldehyde. After fixing, cells were permeabilised in $0.1 \%$ Triton X-100 in PBS, followed by quenching in $50 \mathrm{mM}$ $\mathrm{NH}_{4} \mathrm{Cl}$ in PBS. Cells were blocked in $0.2 \%$ gelatin for $30 \mathrm{~min}$ and subsequently incubated with an $\alpha$-Crm1 primary antibody (1:100 dilution, sc-5595; Santa Cruz Biotechnology) for 45 min in a humidified chamber. After washing in PBS, Cy3-conjugated 
goat anti-rabbit antibody (1:300; Jackson ImmunoResearch Laboratories, West Grove, PA, USA) was applied for a further $45 \mathrm{~min}$. Cell nuclei were stained with DAPI (100 ng/ml) and coverslips were mounted in Mowiol. Fluorescence was visualised using standard fluorescence microscopy.

Cell proliferation assay. To examine the effect of Crm1 siRNA or LMB on cell viability, the MTT assay was used. For the analysis of the effect of Crm1 siRNA on cell viability, $2.0 \times 10^{3}$ cells were seeded into each well of a 96-well plate and transfected with $20 \mathrm{nM}$ siRNA on the following day, whereas for treatment with LMB, 1.0x $10^{4}$ cells were seeded into each well of a 96-well plate and treated with varying concentrations of LMB on the following day. Cell proliferation was measured five days after siRNA transfection and two days after LMB treatment by the addition of MTT reagent (Sigma), and crystals were subsequently solubilized using solubilization buffer. Absorbance was measured at an OD of $595 \mathrm{nM}$ using a microplate reader.

Cell cycle analysis. For analysis of the effect of Crm1 inhibition on the cell cycle, $1.8 \times 10^{5} \mathrm{KYSE} 30$ and WHCO5 cells were plated on $60-\mathrm{mm}$ dishes and transfected with $20 \mathrm{nM}$ control or Crml siRNA for $72 \mathrm{~h}$. Subsequently, cells were harvested and fixed in $95 \%$ ethanol, after which the cells were stained with propidium iodide and the cell cycle profiles were analyzed using the BD FACSVerse ${ }^{\mathrm{TM}}$ flow cytometer (BD Biosciences, Franklin Lakes, NJ, USA). Quantification of the percentage of cells at different stages of the cell cycle was performed using Modfit 3.3 software.

Caspase-3/7 assay. To assay for caspase-3/7 activity, the Caspase-Glo ${ }^{\mathrm{TM}}$ 3/7 assay (Promega) was performed, according to the manufacturer's instructions. Briefly, $2.0 \times 10^{3}$ cells were plated/well in 96-well plates and transfected with $20 \mathrm{nM}$ control or Crm1 siRNA. Caspase-3/7 activity was measured $48 \mathrm{~h}$ after siRNA transfection, and luminescence was monitored using the Veritas ${ }^{\mathrm{TM}}$ microplate luminometer (Promega). MTT assays were performed concurrently and caspase-3/7 luminescence values were normalised to OD595 values to control for any differences in cell number.

Statistical analysis. The statistical significance of the means was calculated using the Student's t-test. Fisher's exact tests were used to compare the expression of Crm1 in the different groups. Survival analysis was carried out using the Kaplan-Meier method. A p-value of $<0.05$ was required for statistical significance.

\section{Results}

Crml immunostaining in normal and tumour tissues of the oesophagus. Altered Crm1 expression has been described in certain cancer types. In the present study we investigated Crm1 expression in oesophageal squamous cell carcinoma (OSCC). Crm1 expression was investigated in formalin-fixed tissue sections of matched tumour and normal stratified squamous epithelium, obtained from 56 South African patients with oesophageal cancer. The basic patient demographics and pathological characteristics are documented in Table I. Among
Table I. Patient demographics.

Characteristics No. of patients $(\%)$

Gender
Male
Female
Age (years)
Average
Range
Race
Black

Mixed ancestry

Caucasian

Smoker

Yes

No

Alcohol intake

Heavy

Light

Unknown

Stage

I

II

III

Differentiation status

Well

Moderate

Poor

Unknown

Presence of keratinisation

Keratinisation

No keratinisation

our patient cohort, $4 \%$ had stage I disease, $55 \%$ had stage II disease and $41 \%$ had stage III disease, based on the TNM (tumour-node-metastasis) staging system (Table I). Due to the late stage at which oesophageal carcinoma is diagnosed, this distribution was as expected.

Immunohistochemical analysis, followed by independent scoring by a pathologist, revealed that most normal sections had weak nuclear staining for Crm1, with no observable cytoplasmic staining (Fig. 1A-C). The corresponding cancer sections, however, displayed much more intense nuclear staining, as represented in Fig. 1D, with $82 \%$ of the patient specimens displaying elevated nuclear staining in the cancer tissues compared to the paired normal tissues. It was also noted that the cancer specimens displayed a much more intense cytoplasmic and nuclear membrane staining compared to the paired normal sections (Fig. 1E and F). Fig. 1A-C shows normal and cancer sections from representative patients with stage I, II and III cancer, showing intense nuclear and cytoplasmic staining of $\mathrm{Crm} 1$ in all three cancer stages, compared 


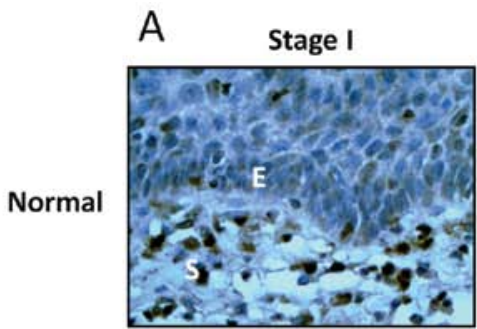

B Stage II
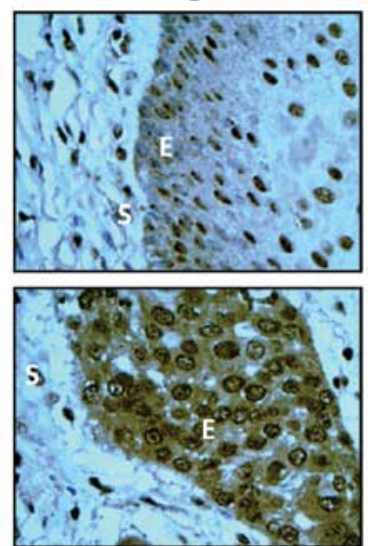

D

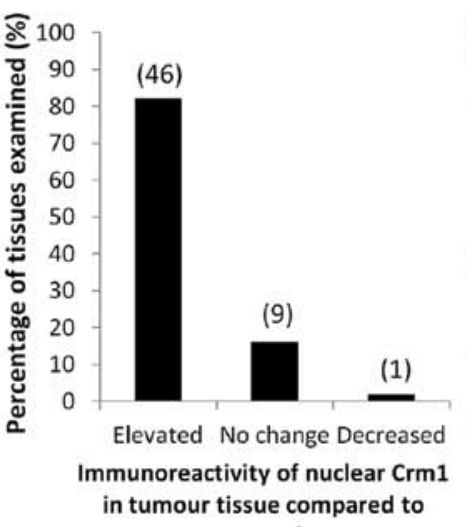
normal
E

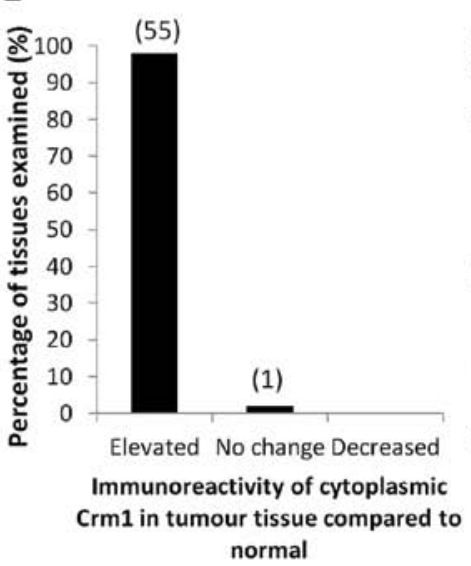

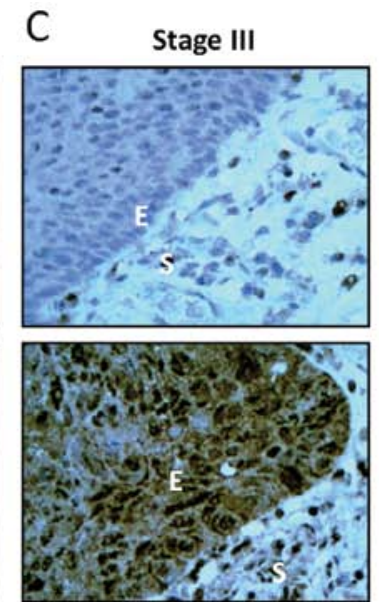

$\mathrm{F}$

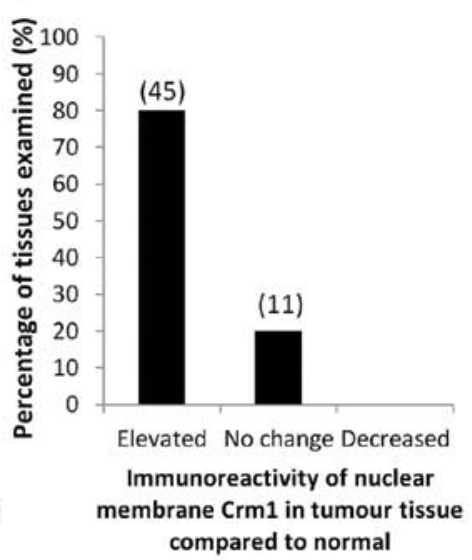

G

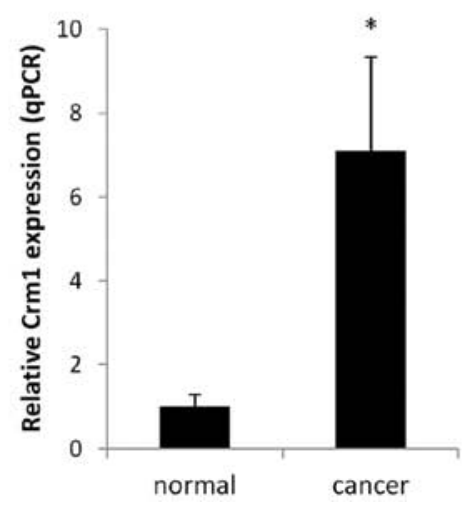

Figure 1. Immunohistochemical analysis of Crm1 expression in normal and tumour tissue sections from patients with oesophageal squamous cell carcinoma. (A-C) Crm1 staining patterns in the different tumour stages are shown. Little immunoreaction of Crm1 was observed in normal oesophageal epithelium. In contrast, intense nuclear and cytoplasmic expression of Crm1 was observed in the tumour tissue (magnification, x40). E, epithelium; S, stroma. (D-F) Elevated Crm1 expression in the tumour tissue compared to the normal tissues was observed in the nuclear (D), cytoplasmic (E) and nuclear membrane (F) cellular compartments. (G) Crm1 mRNA levels in matched normal and tumour tissues obtained from 22 patients with OSCC ( ${ }^{*}$ p $\left.<0.05\right)$. Results are shown as the mean \pm SEM. Crm1, the chromosome region maintenance 1 protein or exportin 1.

to their corresponding normal tissues. A statistical analysis revealed that the difference in staining pattern between normal and cancer specimens was statistically significant for all staining categories (nuclear, cytoplasmic and nuclear membrane staining) (Table II).

It was next determined whether any correlation exists between the expression of $\mathrm{Crm} 1$ and tumour stage. Correlation analyses were performed and demonstrated that the expression of Crm1 (where nuclear, cytoplasmic and nuclear membrane staining patterns were taken into account) did not significantly associate with tumour stage (Table III). Since a similar proportion of stage II and stage III patients displayed strong Crm1 expression, it was suggested that the increase in expression of $\mathrm{Crm} 1$ is a relatively early event in oesophageal carcinogenesis (occurring by the time the cancer has progressed to stage II), rather than a late stage event. 
Table II. Expression of Crm1 in normal and cancer tissue of the oesophagus.

\begin{tabular}{|c|c|c|c|}
\hline \multirow[b]{2}{*}{ Crm1 expression } & \multicolumn{2}{|c|}{ No. of patients (\%) } & \multirow[b]{2}{*}{ P-value ${ }^{a}$} \\
\hline & Normal & Cancer & \\
\hline \multicolumn{4}{|l|}{ Nuclear staining } \\
\hline Negative & $8(14)$ & $3(5)$ & \\
\hline Weak & $48(86)$ & $8(14)$ & $<0.0001$ \\
\hline Strong & $0 \quad(0)$ & $45(80)$ & \\
\hline \multicolumn{4}{|c|}{ Cytoplasmic staining } \\
\hline Negative & $55(98)$ & $2(4)$ & \\
\hline Weak & $1(2)$ & $26(46)$ & $<0.0001$ \\
\hline Strong & $0 \quad(0)$ & $28(50)$ & \\
\hline \multicolumn{4}{|c|}{ Nuclear membrane staining } \\
\hline Negative & $55(98)$ & $10(18)$ & \\
\hline Weak & 1 (2) & $41(73)$ & $<0.0001$ \\
\hline Strong & $0 \quad(0)$ & $5 \quad(9)$ & \\
\hline
\end{tabular}

${ }^{\mathrm{a}}$ Fisher's exact test.

Since Crm1 levels were found to be significantly elevated in oesophageal tumour tissues in both the cytoplasmic and nuclear cellular components, a Kaplan-Meier survival analysis was performed to determine whether $\mathrm{Crm} 1$ expression predicts patient survival. A trend, where high cytoplasmic Crm1 expression appeared to be associated with poor overall survival, was observed (data not shown). There was no correlation between nuclear Crm1 expression and overall survival. These findings were likely influenced by the limited data and tissue specimens available for early stage oesophageal cancer patients. Access to early stage patient material may have a significant effect on the interpretation of overall survival analyses.

Crml mRNA expression in patient tissues. As an increase in Crm1 protein expression was observed in oesophageal cancer patient specimens, it was next investigated whether Crm1 was similarly upregulated at the mRNA level. Crm1 mRNA levels were thus examined by quantitative real-time RT-PCR using RNA obtained from matched normal and cancer tissue biopsies obtained from 22 patients with OSCC. A significant increase in Crm1 mRNA expression was observed in the oesophageal tumour specimens compared to the normal tissues (Fig. 1G), suggesting that the increase in Crm1 protein expression observed by IHC was derived from elevated $\mathrm{Crm} 1$ mRNA expression.

Expression of Crml in normal and cancer oesophageal cell lines. As elevated Crm1 expression was observed in oesophageal cancer tissues compared to that in the normal tissues, Crm1 expresssion was next evaluated in 9 oesophageal cancer cell lines (from South African and Japanese origin) and compared to the expression in hTERT-immortalised EPC2 cells derived from normal oesophageal epithelium. Western blot analysis revealed that $\mathrm{Crm} 1$ protein expression was high in all the oesophageal cancer cell lines (WHCO1, WHCO5, WHCO6, KYSE30, KYSE70, KYSE150, KYSE180, KYSE420, KYSE450), while lowest expression was observed in the

Table III. Relationship between Crm1 expression and tumour stage.

\begin{tabular}{|c|c|c|c|c|}
\hline \multirow[b]{2}{*}{ Crm1 expression } & \multicolumn{3}{|c|}{ No. of patients $(\%)$} & \multirow[b]{2}{*}{ P-value } \\
\hline & Stage I & Stage II & Stage III & \\
\hline \multicolumn{5}{|l|}{ Nuclear expression } \\
\hline Negative & $0 \quad(0)$ & $3(10)$ & $0 \quad(0)$ & \\
\hline Weak & $1 \quad(50)$ & $4(13)$ & $4(17)$ & 0.200 \\
\hline Strong & $1 \quad(50)$ & $24(77)$ & $19(83)$ & \\
\hline \multicolumn{5}{|c|}{ Cytoplasmic expression } \\
\hline Negative & $0 \quad(0)$ & $2(6)$ & $0 \quad(0)$ & \\
\hline Weak & $2(100)$ & $13(42)$ & $11(48)$ & 0.437 \\
\hline Strong & $0 \quad(0)$ & $16(52)$ & $12(52)$ & \\
\hline \multicolumn{5}{|c|}{ Nuclear membrane expression } \\
\hline Negative & $0 \quad(0)$ & $7(23)$ & $3(13)$ & \\
\hline Weak & $2(100)$ & $22(71)$ & $18(78)$ & 0.679 \\
\hline Strong & $0 \quad(0)$ & $2(6)$ & $2(9)$ & \\
\hline \multicolumn{5}{|l|}{ Overall expression } \\
\hline Weak & $1 \quad(50)$ & $6(19)$ & $2(9)$ & \\
\hline Moderate & $1 \quad(50)$ & $15(48)$ & $11(48)$ & 0.184 \\
\hline Strong & $0 \quad(0)$ & $10(32)$ & $10(43)$ & \\
\hline
\end{tabular}

${ }^{\mathrm{a}}$ Fisher's exact test. 
A

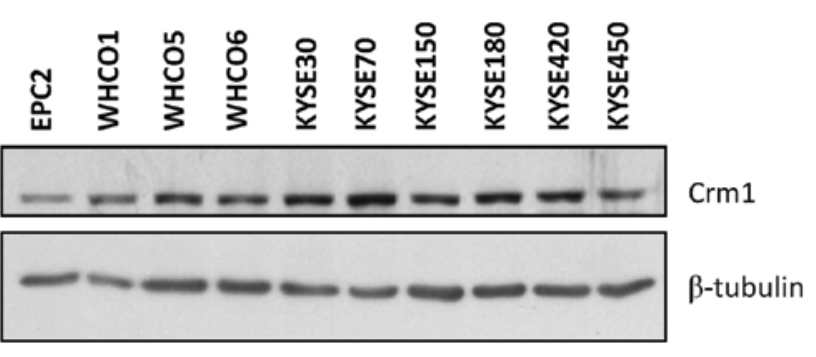

B

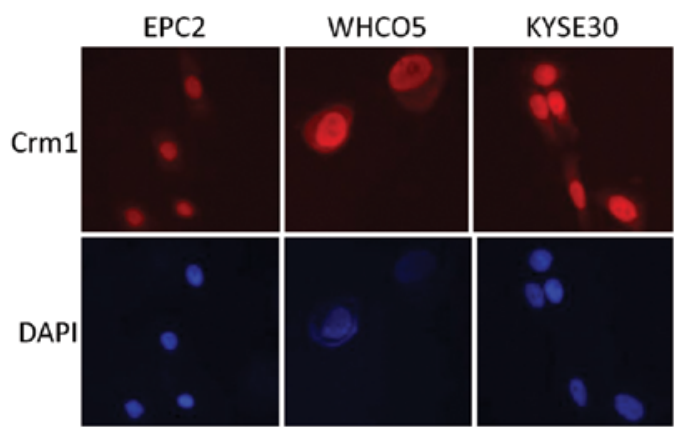

$\mathbf{E}$

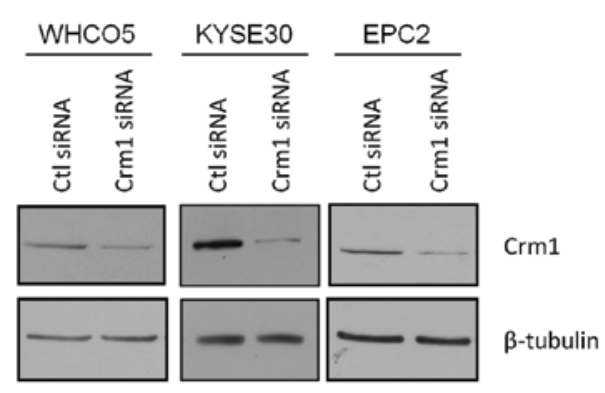

C

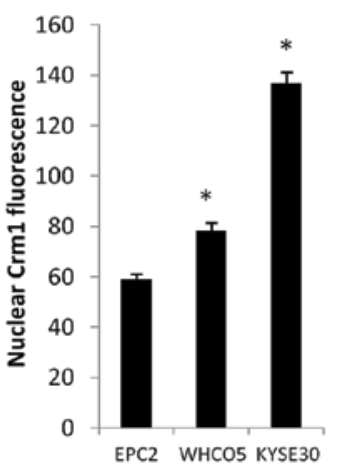

F
D
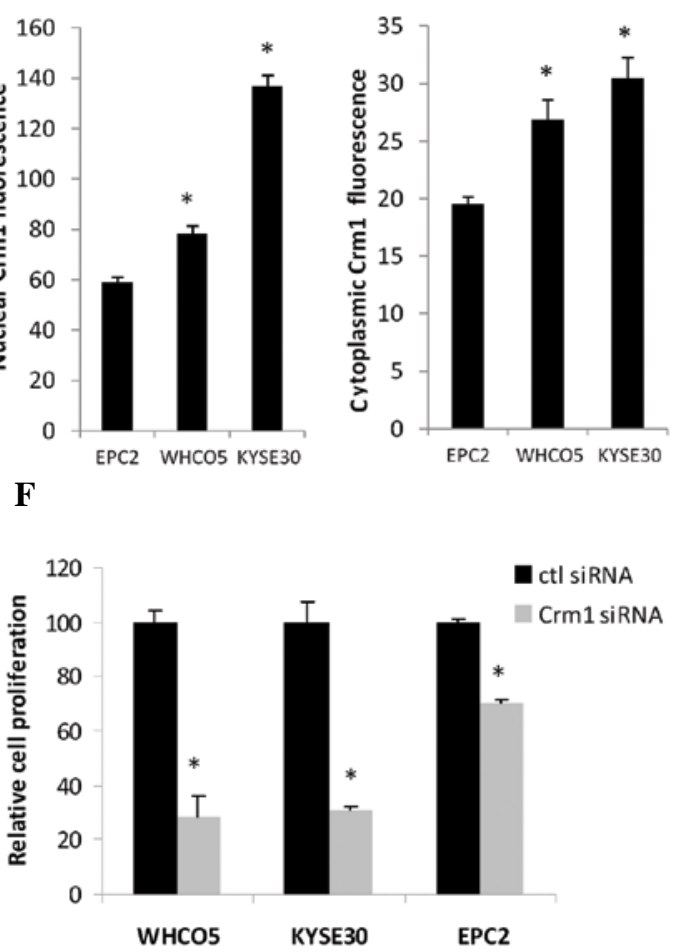

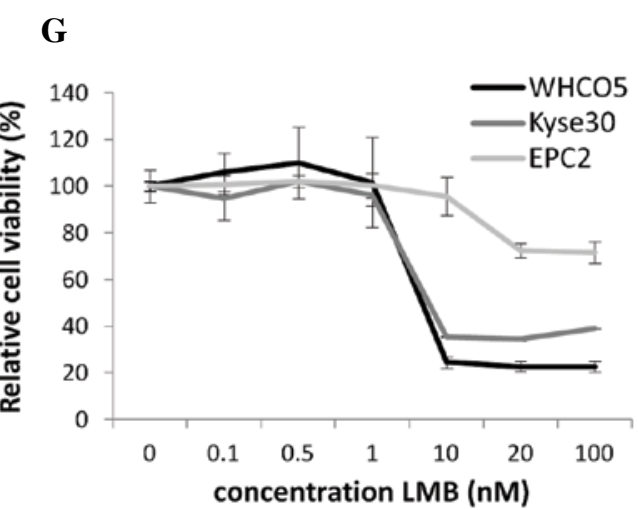

Figure 2. Crm1 expression is elevated in cultured oesophageal cancer cell lines and is necessary for cancer cell survival. (A) Western blot analysis showing Crm1 protein levels in normal immortalised oesophageal epithelial EPC2 cells and in oesophageal cancer cell lines. $\beta$-tubulin was used as a loading control. (B) Immunofluorescence showing Crm1 localisation in normal EPC2 cells and representative cancer cells, WHCO5 and KYSE30. DAPI staining shows the cell nuclei. (C and D) Quantitation of Crm1 fluorescence intensity in the nucleus (C) and cytoplasm (D) of approximately 40 cells stained with the Crm1 antibody reveals a significant increase in Crm1 expression in the cancer cell lines compared to normal EPC2 cells ( $\mathrm{p}<0.05)$. (E) Western blot analysis showing a reduction in Crm1 protein levels after transfection of WHCO5, KYSE30 and EPC2 cell lines with Crm1 siRNA, compared to control (ctl) siRNA. (F) MTT assay showing a more pronounced decrease in proliferation of WHCO5 and KYSE30 oesophageal cancer cells after Crm1 knockdown, compared to non-cancer EPC2 cells ("p<0.05); (G) Cell viability curves showing the response of WHCO5, KYSE30 and EPC2 cells to varying concentrations of the $\mathrm{Crm1}$ inhibitor, leptomycin B. Experiments were performed in triplicate and repeated at least two times. Results are shown as the mean \pm SEM. Crm1, the chromosome region maintenance 1 protein or exportin 1.

normal epithelial cell line, EPC2 (Fig. 2A). Moreover, Crm1 localisation in the normal and representative cancer (WHCO5 and KYSE30) cell lines was determined by immunofluorescence, and quantitation revealed that there was a significant 
$\mathbf{A}$

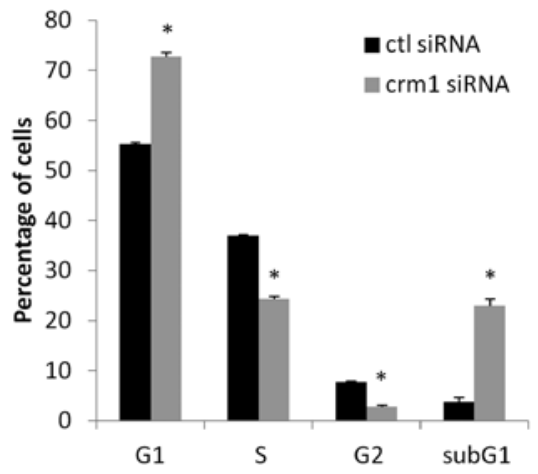

B

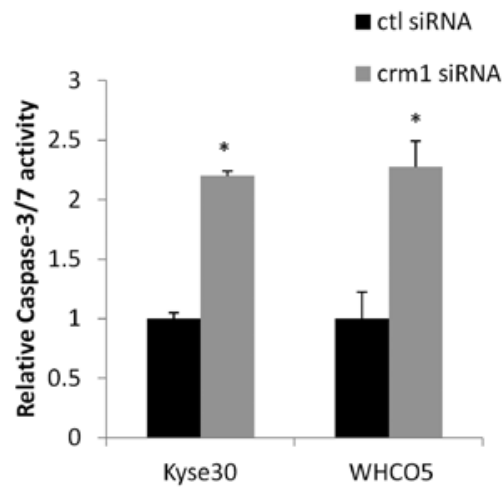

Figure 3. Inhibition of Crm1 expression in oesophageal cancer cells results in cell cycle arrest and the induction of apoptosis. (A) Effect of Crm1 siRNA on cell cycle progression. KYSE30 cells were transfected with $20 \mathrm{nM}$ control siRNA or Crm1 siRNA and harvested $72 \mathrm{~h}$ later for cell cycle analysis by flow cytometry. Crm1-knockdown cells exhibited G1 cell cycle arrest and the induction of the subG1 population. Similar results were found using the WHCO5 cell line ( $\left.{ }^{*} \mathrm{p}<0.05\right)$. (B) Caspase-3/7 activity was measured $48 \mathrm{~h}$ after transfection of cells with $20 \mathrm{nM}$ control or Crm1 siRNA. A significant increase in caspase-3/7 activity was observed after Crm1 knockdown in both KYSE30 and WHCO5 cell lines. Experiments were performed in triplicate and repeated at least two times $(\mathrm{p}<0.05)$. Results are shown as the mean \pm SEM.

increase in $\mathrm{Crm} 1$ fluorescence in the cancer cell lines, in both the nuclear and cytoplasmic compartments (Fig. 2B-D). These results are in agreement with the patient data showing elevated nuclear and cytoplasmic Crm1 expression in oesophageal tumour tissues compared to normal tissues.

Effect of Crml inhibition on cell survival. To evaluate the functional significance of elevated Crm1 expression in oesophageal cancer, Crm1 siRNA was used to inhibit its expression. A control siRNA with no known silencing effect was used to control for the non-specific effects of siRNA transfection. WHCO5 and KYSE30 oesophageal cancer cells and EPC2 normal oesophageal cells were transfected with $20 \mathrm{nM}$ siRNA, and protein knockdown was confirmed by western blot analysis (Fig. 2D). The effect of Crm1 siRNA on cell proliferation was next determined. As shown in Fig. 2E, a significant reduction in oesophageal cancer cell proliferation was noted five days after siRNA transfection. The effect of Crm1 knockdown on EPC2 cell proliferation, on the other hand, was much less pronounced (Fig. 2E).

To corroborate the effect of Crm1 siRNA on oesophageal cancer cell proliferation, the commercially available $\mathrm{Crm} 1$ inhibitor, leptomycin B (LMB), was used. Cells were treated with varying concentrations of LMB, and cell proliferation was determined $48 \mathrm{~h}$ later. In line with the siRNA data, WHCO5 and KYSE30 cancer cells were significantly more sensitive to LMB treatment (70-80\% decrease in cell number at $100 \mathrm{nM} \mathrm{LMB}$ ) when compared to the normal EPC2 cells, which showed little cytotoxicity, even at high LMB concentrations (30\% decrease in cell number at $100 \mathrm{nM}$ LMB) (Fig. 2F).

These results suggest that $\mathrm{Crm} 1$ expression is upregulated in oesophageal cancer cell lines and that these cells are highly dependent on its expression for cell survival and proliferation, whereas non-cancer oesophageal cells appear less reliant on its expression.

Effect of Crm1 inhibition on the cell cycle and apoptosis. Since Crm1 inhibition reduced oesophageal cancer cell proliferation, we next investigated whether $\mathrm{Crm} 1$ inhibition associated with alterations in cell cycle distribution. Crm1 expression was inhibited in KYSE30 oesophageal cancer cells transfected with siRNA for $72 \mathrm{~h}$. FACS analysis revealed that the inhibition of $\mathrm{Crm} 1$ resulted in a significant increase in cell in G1, associated with a significant decrease in the number of cells in the $\mathrm{S}$ and G2/M phases of the cell cycle (Fig. 3A). In addition, $\mathrm{Crm} 1$ inhibition resulted in the induction of a subG1 population, indicative of apoptosis. Similar results were obtained using the WHCO5 oesophageal cancer cell line (data not shown). To confirm that $\mathrm{Crm} 1$ inhibition induces apoptosis in oesophageal cancer cells, a caspase-3/7 assay was performed where caspase-3/7 activity was measured in KYSE30 and WHCO5 cells after transfection with control or Crm1 siRNA. Fig. 3B shows a significant increase in caspase-37 activity in cells transfected with Crm1 siRNA compared to control siRNA-transfected cells, confirming the induction of apoptosis after $\mathrm{Crm} 1$ inhibition.

\section{Discussion}

This study investigated the expression of the nuclear transport receptor, Crm1, in oesophageal cancer. Crm1 showed weak nuclear expression patterns in normal oesophageal epithelium, while its expression was much stronger and localised to the nucleus, cytoplasm and nuclear envelope in oesophageal tumour tissues. Real-time RT-PCR analysis revealed that Crm1 was also elevated at the mRNA level. Western blot analysis confirmed the elevated expression of Crm1 in oesophageal cancer cells compared to normal oesophageal epithelial cells. $\mathrm{Crm} 1$ has recently been reported to be expressed at elevated levels in cervical squamous cell carcinoma, ovarian carinoma, osteosarcoma, glioma, pancreatic cancer, gastric cancer and multiple myeloma (7-13). To the best of our knowledge, this is the first study reporting elevated expression of $\mathrm{Crm} 1$ in oesophageal cancer.

It is likely that the elevated levels of $\mathrm{Crm} 1$ in tumour tissues are derived from its cell cycle-dependent regulation. Evidence suggests that $\mathrm{Crm} 1$ is a cell cycle-regulated gene, as its mRNA levels have been reported to oscillate with the cell cycle, where Crm1 expression is initiated in late G1 and reaches a peak at G2/M (22). We recently reported that the $\mathrm{Crm} 1$ promoter is regulated by NFY and Sp1, both transcription factors that function in concert with the cell cycle and are found at elevated 
levels in transformed and cancer cells (23). Furthermore, we found that p53 represses the $\mathrm{Crm} 1$ promoter in response to DNA damage (23), also likely contributing to elevated Crm1 in cancer cells, as most cancer cells either contain low levels of wild-type p53 protein (through enhanced p53 degradation) or harbor p53 mutations, including oesophageal cancer $(24,25)$. The regulation of Crm1 expression by NFY, Sp1 and p53 transcription factors in cancer cells likely results in an increased expression of Crm1 compared to that in normal cells.

We report here that the nuclear, cytoplasmic and nuclear membrane expression of $\mathrm{Crm} 1$ is increased in oesophageal tumours. This localisation pattern is in line with Noske et al, who observed an increase in both nuclear and cytoplasmic Crm1 staining in ovarian carcinomas, when compared to normal tissues (8). Notably, however, Shen et al showed that Crm1 expression in gliomas was predominantly nuclear, with only weak cytoplasmic immunoreaction (10), suggesting that the localization pattern of Crm1 does vary with tumour type. Both studies made use of the same antibody to stain for Crm1 expression as in the present study. Furthermore, we report that there is a trend where high Crm1 expression associates with poor patient survival, supporting studies showing a prognostic role for Crm1 in other cancer types (8-11). However, due to the limited sample size of early stage disease, prospective large-scale studies are required to investigate the true prognostic role of high Crm1 expression in human oesophageal squamous cell carcinoma.

While the prognostic value of elevated Crm1 in oesophageal cancer has not yet been determined conclusively, our findings suggest that it may have value as an oesophageal cancer therapeutic target. Our results demonstrate a role for Crm1 in the biology of oesophageal cancer as its inhibition resulted in G1 cell cycle arrest and the induction of apoptosis. We similarly found that treatment with the Crm1 inhibitor, leptomycin B, resulted in oesophageal cancer cell death. Interestingly, normal oesophageal epithelial cells were less sensitive to Crm1 inhibition using siRNA and LMB, suggesting that targeting Crm 1 could be an attractive therapy for oesophageal cancer. Other studies have similarly reported an increased sensitivity of cancer cells to Crm1 inhibition compared to their normal counterparts $(7,26,27)$, thus highlighting the need for the development of further Crm1 inhibitors. It is interesting to note that $\mathrm{Crm} 1$ overexpression appears to be necessary for the therapeutic efficacy of drugs targeting Crm1 as cells with low Crm1 expression, for example normal cells, are less sensitive to Crm1 inhibition. The increased reliance of cancer cells on Crm1 compared to normal cells is likely due to the increased nuclear transport rates in cancer cells and thus an increased reliance on the nuclear transport machinery. This is supported by a recent study by Kuusisto et al who reported that nuclear import and export activity is increased in transformed cells compared to non-transformed cells due to increased expression of the importer and exporter proteins, respectively (28).

Overall, we demonstrated that oesophageal cancer cells contain elevated levels of $\mathrm{Crm} 1$ protein and are highly reliant on its expression, as its inhibition results in cancer cell death, while in normal cells its inhibition has only a minor effect. Based on this, we propose that Crm1 has potential as a diagnostic marker and/or therapeutic target for patients with oesophageal squamous cell carcinoma. Further study however is required in order to clarify its true clinical significance.

\section{Acknowledgements}

We thank Ms. Antionette Olivier for providing patient information. This study was supported by grants from the Cancer Association of South Africa (CANSA), the University of Cape Town and the Carnegie Corporation of New York.

\section{References}

1. Parkin DM, Bray F, Ferlay J and Pisani P: Global cancer statistics, 2002. CA Cancer J Clin 55: 74-108, 2005.

2. Jemal A, Bray F, Center MM, Ferlay J, Ward E and Forman D: Global cancer statistics. CA Cancer J Clin 61: 69-90, 2011.

3. Ossareh-Nazari B, Bachelerie F and Dargemont C: Evidence for a role of CRM1 in signal-mediated nuclear protein export. Science 278: 141-144, 1997.

4. Fukuda M, Asano S, Nakamura T, Adachi M, Yoshida M, Yanagida M and Nishida E: CRM1 is responsible for intracellular transport mediated by the nuclear export signal. Nature 390: 308-311, 1997.

5. Stommel JM, Marchenko ND, Jimenez GS, Moll UM, Hope TJ and Wahl GM: A leucine-rich nuclear export signal in the p53 tetramerization domain: regulation of subcellular localization and p53 activity by NES masking. EMBO J 18: 1660-1672, 1999.

6. Saji M, Vasko V, Kada F, Allbritton EH, Burman KD and Ringel MD: Aktl contains a functional leucine-rich nuclear export sequence. Biochem Biophys Res Commun 332: 167-173, 2005.

7. van der Watt PJ, Maske CP,Hendricks DT, et al: The Karyopherin proteins, Crm1 and Karyopherin $\beta 1$, are overexpressed in cervical cancer and are critical for cancer cell survival and proliferation. Int J Cancer 124: 1829-1840, 2009.

8. Noske A, Weichert W, Niesporek S, et al: Expression of the nuclear export protein chromosomal region maintenance/exportin 1/Xpol is a prognostic factor in human ovarian cancer. Cancer 112: 1733-1743, 2008.

9. Yao Y, Dong Y, Lin F, et al: The expression of CRM1 is associated with prognosis in human osteosarcoma. Oncol Rep 21: 229-235, 2009

10. Shen A, Wang Y, Zhao Y, Zou L, Sun L and Cheng C: Expression of CRM1 in human gliomas and its significance in p27 expression and clinical prognosis. Neurosurgery 65: 153-159, 2009.

11. Huang WY, Yue L, Qiu WS, Wang LW, Zhou XH and Sun YJ: Prognostic value of CRM1 in pancreas cancer. Clin Invest Med 32: E315, 2009

12. Zhou F, Qiu W, Yao R, et al: CRM1 is a novel independent prognostic factor for the poor prognosis of gastric carcinomas. Med Oncol 30: 726, 2013.

13. Tai YT, Landesman Y, Acharya C, et al: CRM1 inhibition induces tumor cell cytotoxicity and impairs osteoclastogenesis in multiple myeloma: molecular mechanisms and therapeutic implications. Leukemia 28: 155-165, 2014.

14. Roberts BJ, Hamelehle KL, Sebolt JS and Leopold WR: In vivo and in vitro anticancer activity of the structurally novel and highly potent antibiotic CI-940 and its hydroxy analog (PD 114,721). Cancer Chemother Pharmacol 16: 95-101, 1986.

15. Mutka SC, Yang WQ, Dong SD, Ward SL, Craig DA, Timmermans PB and Murli S: Identification of nuclear export inhibitors with potent anticancer activity in vivo. Cancer Res 69: 510-517, 2009.

16. Van Neck T, Pannecouque C, Vanstreels E, Stevens M, Dehaen W and Daelemans D: Inhibition of the CRM1-mediated nucleocytoplasmic transport by $\mathrm{N}$-azolylacrylates: structure-activity relationship and mechanism of action. Bioorg Med Chem 16: 9487-9497, 2008.

17. Allred DC, Brown P and Medina D: The origins of estrogen receptor $\alpha$-positive and estrogen receptor $\alpha$-negative human breast cancer. Breast Cancer Res 6: 240-245, 2004.

18. Maae E, Nielsen M, Steffensen KD, Jakobsen EH, Jakobsen A and Sorensen FB: Estimation of immunohistochemical expression of VEGF in ductal carcinomas of the breast. J Histochem Cytochem 59: 750-760, 2011. 
19. Livak KJ and Schmittgen TD: Analysis of relative gene expression data using real-time quantitative PCR and the $2^{-\Delta \Delta C \mathrm{~T}}$ method. Methods 25: 402-408, 2001.

20. Jones GJ, Heiss NS, Veale RB and Thornley AL: Amplification and expression of the TGF- $\alpha$, EGF receptor and c-myc genes in four human oesophageal squamous cell carcinoma lines. Biosci Rep 13: 303-312, 1993.

21. Shimada $\mathrm{Y}$, Imamura $\mathrm{M}$, Wagata $\mathrm{T}$, Yamaguchi $\mathrm{N}$ and Tobe $\mathrm{T}$ Characterization of 21 newly established esophageal cancer cell lines. Cancer 69: 277-284, 1992.

22. Kudo N, Khochbin S, Nishi K, Kitano K, Yanagida M, Yoshida M and Horinouchi S: Molecular cloning and cell cycle-dependent expression of mammalian CRM1, a protein involved in nuclear export of proteins. J Biol Chem 272: 29742-29751, 1997.

23. van der Watt PJ and Leaner VD: The nuclear exporter, Crm1, is regulated by NFY and Sp1 in cancer cells and repressed by p53 in response to DNA damage. Biochim Biophys Acta 1809: 316-326, 2011.
24. Casson AG, Tammemagi M, Eskandarian S, Redston M, McLaughlin J and Ozcelik H: p53 alterations in oesophageal cancer: association with clinicopathological features, risk factors, and survival. Mol Pathol 51: 71-79, 1998.

25. Uchino S, Saito T, Inomata M, Osawa N, Chikuba K, Etoh K and Kobayashi M: Prognostic significance of the p53 mutation in esophageal cancer. Jpn J Clin Oncol 26: 287-292, 1996.

26. Lecane PS, Kiviharju TM, Sellers RG and Peehl DM Leptomycin B stabilizes and activates p53 in primary prostatic epithelial cells and induces apoptosis in the $\mathrm{LNCaP}$ cell line. Prostate 54: 258-267, 2003.

27. Smart P, Lane EB, Lane DP, Midgley C, Vojtesek B and Lain S: Effects on normal fibroblasts and neuroblastoma cells of the activation of the p53 response by the nuclear export inhibitor leptomycin B. Oncogene 18: 7378-7386, 1999.

28. Kuusisto HV, Wagstaff KM, Alvisi G, Roth DM and Jans DA: Global enhancement of nuclear localization-dependent nuclear transport in transformed cells. FASEB J 26: 1181-1193, 2011. 